\title{
Docentes frente al covid-19: cambios percibidos en Chile y Perú
}

Teachers Facing Covid-19: Perceived Changes in Chile and Peru

Artículo de investigación | Research article

Fecha de recepción: 05 de diciembre de 2020

Fecha de aceptación: 26 de abril de 2021

Fecha de disponibilidad en línea: octubre de 2021

doi: 10.11144/Javeriana.m14.dfcc

Julio-César Mateus

JMATEUS@ULIMA.EDu.PE

Universidad de Lima, Perú

ORCID: https://orcid.org/0000-0001-5161-3737

Pablo Andrada pablo.andrada@userena.cl

Universidad de La Serena, Chile

ORCID: https://orcid.org/0000-0002-2887-5517

Para citar este artículo | To cite this article

Mateus, J. C. \& Andrada, P. (2021). Docentes frente al covid-19: cambios percibidos en Chile y Perú. magis, Revista Internacional de Investigación en Educación, 14, 1-25. doi: 10.11144/Javeriana.m14.dfcc 


\title{
Resumen
}

Este artículo de investigación busca describir la percepción de los docentes sobre sus condiciones y los cambios en sus prácticas pedagógicas a partir de la emergencia sanitaria causada por el covid-19. Participaron del estudio una muestra de 541 profesores chilenos y peruanos de todos los niveles educativos (preescolar, primaria, secundario y superior) a través de un cuestionario estructurado virtual. Los hallazgos confirman el impacto de las desigualdades sociales, demográficas e históricas presentes en ambos países.

\section{Palabras clave}

Docente; papel del docente; actitud del docente; competencias del docente; condiciones de empleo del docente; educación a distancia

\begin{abstract}
This research seeks to describe the teachers' perceptions about their conditions and the changes experienced in their pedagogical practices due to the health emergency caused by Covid-19. A sample of 541 Chilean and Peruvian teachers of all educational levels participated in the study (preschool, primary, secondary and higher ed), through a structured virtual questionnaire. The findings confirmed the impact of the social, demographic and historical inequalities present in both countries.
\end{abstract}

\section{Keywords}

Teachers; teacher role; teacher attitudes; teacher qualifications; teacher conditions of employment; distance education 
Descripción del artículo | Article description

Este artículo de investigación forma parte del proyecto internacional eduCOVID19. Research on how the COVID19 pandemic changed the educational scenario.

\section{Introducción}

La transformación digital de las instituciones educativas latinoamericanas previo a la emergencia sanitaria del covid-19 se cocinaba a fuego lento. Si bien América Latina era reconocida como una región dinámica que invertía en compra de tecnología, las brechas en calidad, infraestructura, acceso y conectividad, así como en el desarrollo de capacidades docentes para integrar las TIC seguían siendo retos pendientes. La nueva "pedagogía pandémica" (Giroux et al., 2020) nos permite poner sobre la mesa el valor de estas brechas, así como actualizar el debate sobre el sentido de los sistemas educativos y la agencia docente en un contexto adverso.

Este trabajo forma parte del proyecto de investigación internacional EduCovid19, cuya finalidad es explorar la percepción de los docentes sobre sus condiciones y los cambios en sus prácticas pedagógicas a partir de la emergencia sanitaria causada por el coronavirus. En este estudio aportamos evidencia empírica de la opinión de los docentes de todos los niveles educativos (preescolar, primario, secundario y superior) en los contextos chileno y peruano.

\section{Brechas estructurales y de acceso a las TIC en la región}

Para Giroux et al. (2020), este contexto de pandemia "y el consiguiente freno total de la actividad presencial de todos los centros educativos, no ha hecho más que encender un reflector gigante que ilumina las desigualdades sociales preexistentes, entre las que destacan los descomunales niveles de segregación escolar" (p. 4). América Latina es la región con mayores brechas del mundo y el Banco Interamericano de Desarrollo (BID) alude a un contrato social fracturado para referirse a ellas.

Al respecto, hasta el momento han sido ampliamente abordadas las brechas entre los sectores rurales y urbanos o entre las instituciones públicas y privadas. A estas diferencias se suman las de género: en América Latina las mujeres trabajan más por un salario menor.

La segmentación extrema hace que un alumno latinoamericano tenga una probabilidad seis veces más alta de educarse con pares de su mismo estatus socioeconómico; mientras que en la OCDE el promedio de este 
indicador es 3, en Chile y Perú la ratio se acerca a 10. Más del $40 \%$ de la matriculación en la escuela secundaria corresponde a escuelas privadas (contra un $10 \%$ en los países de la OCDE). En ese contexto, los estudiantes ricos tienen más posibilidades de tener un docente con posgrado, mientras que es más factible que los más pobres tengan docentes con menos de 5 años de experiencia (Busso \& Messina, 2020).

Asimismo, las pruebas de calidad que se aplican en cada país permiten observar la correlación directa y sostenida en las últimas décadas entre resultados y grupos socioeconómicos. Si bien estas disminuyen, lo hacen sin alterar las distancias entre sí, es decir, los avances no permiten pensar aún que la educación pueda ofrecer en el corto plazo una esperanza de movilidad social (Arraigada-Toledo, 2020; Cuenca \& Urrutia, 2019).

En cuanto al acceso a TIC, además de teléfonos móviles, el dispositivo más común dentro de los hogares latinoamericanos es la computadora portátil, con un promedio de 57 \% de estudiantes. Según un reciente informe de la CEPAL y la Unesco, "las diferencias según condición socioeconómica y cultural son bastante significativas, en el caso de cada uno de los dispositivos. [...] El acceso a computadoras de escritorio y tabletas es menor" (CEPAL-Unesco, 2020, p. 5). En adición, debemos considerar que es probable que varios miembros del hogar compartan un mismo dispositivo para sus actividades educativas o laborales, lo que hace la brecha aún más amplia.

\section{Respuesta a la emergencia sanitaria en Chile y Perú}

En el caso peruano, ante la suspensión de clases escolares presenciales, el Ministerio de Educación desarrolló la estrategia "Aprendo en Casa" (www.aprendoencasa.pe), un programa multiplataforma con producción de material educativo divulgado a través de radio, televisión e internet. Este servicio de distribución de contenidos de clase debía ser complementado con la preparación de material y seguimiento de los docentes a través de los medios de comunicación que tengan a su disposición (mensajes de texto, WhatsApp, etc.). No obstante, las brechas de acceso a las TIC han jugado en contra. Según un estudio del Ministerio de Educación peruano, un tercio de las familias reclama mejor señal de televisión, radio o internet, y el 46.1 \% piden más ayuda para el acompañamiento de sus hijos (El Comercio, 2020).

A nivel de educación superior, el inicio de clases fue postergado varias veces debido a la falta de infraestructura digital — sobre todo en las instituciones públicas - y de desarrollo de capacidades docentes. Al menos el $70 \%$ de universidades peruanas no habían implementado nunca un 
programa virtual (Figallo et al., 2020). En aquellas instituciones que pudieron iniciar sus programas se identificaron problemas de implementación, vinculados con la carencia de instrumentos de evaluación en línea y la sobrecarga de tareas, debido a la inexperiencia en el manejo de clases virtuales (Vicentini, 2020).

Entre otras consecuencias identificables de la pandemia en la educación básica (primaria y secundaria) destaca la migración de más de 100 mil estudiantes de la educación privada al sistema público, el cierre de al menos 2 mil centros educativos privados, la posibilidad de que miles de estudiantes pierdan el año por falta de acceso o acompañamiento, así como la disminución de no menos del $15 \%$ de la matrícula e importantes indicios de deserción escolar, sobre todo en zonas rurales, reabriendo una herida que había curado bastante bien en los últimos años (Cueto et al., 2020). A nivel superior se estima una caída en la matrícula de institutos y universidades de al menos el $20 \%$, por lo que se han dispuesto ayudas y becas para paliar este escenario negativo. La tasa de deserción universitaria es 6 puntos porcentuales mayor respecto al 2019, atribuibles al contexto de emergencia (en las universidades públicas la tasa de deserción actual es de $9.85 \%$, mientras que en las privadas llega al $22.5 \%$ ) (Alayo Orbegozo, 2020).

En Chile, el sistema educacional se encontraba tensionado por la revuelta social que comenzó el 18 de octubre de 2019. A partir de entonces, en la mayoría de los casos no se han retomado con regularidad las clases en jardines infantiles, colegios ni universidades. En el mes de enero de 2020 la prueba universal de acceso a la educación superior fue boicoteada por los estudiantes secundarios, teniendo que suspenderse en un par de ocasiones. Todo este clima ya había perjudicado las matrículas de algunas universidades, en especial las privadas y de menor prestigio. En marzo, la suspensión de clases se fue dando mientras avanzaban los contagios de covid-19. En algunos establecimientos se logró avanzar un par de semanas con las clases, pero los primeros brotes generaron una primera suspensión de estas, el adelanto de vacaciones de invierno y la suspensión indefinida mientras avanzaba el confinamiento. Se estima a partir de allí que 3.6 millones de estudiantes reciben clases a través de internet, y se espera, según estimaciones del Ministerio de Educación, que durante 2020 unos 80 mil niños y jóvenes hayan dejado el sistema escolar (Diario de Concepción, 2020), lo que significa un aumento del $44 \%$ respecto al año anterior.

Desde septiembre, el Ministerio de Educación ha buscado retomar las clases en los lugares que tienen menos contagios y no dar por perdido el año escolar, aunque se ha encontrado con la resistencia del gremio de profesores y de las familias que prefieren no enviar a sus hijos a clases este año (Cadem, 2020). Las universidades por su parte han permitido que algunas 
asignaturas prácticas realicen sus funciones. En cualquiera de los casos, la mayoría del sector educativo se encuentra en modalidad a distancia. Al concluir el primer semestre de 2020 se estimaban en 9 mil los alumnos que habían dejado el sistema universitario o congelado sus estudios en 17 universidades (Said \& Gálvez, 2020).

El Ministerio de Educación ha dispuesto un sitio web (www.aprendoenlinea.cl) en el que se puede encontrar el currículo priorizado para el año 2020, como forma de apoyar los aprendizajes de los estudiantes en los últimos niveles de educación preescolar, además de los de educación primaria y secundaria. En el caso de las universidades, la mayoría ha continuado sus actividades en modalidad a distancia, postergando algunas asignaturas prácticas de las carreras.

Otra iniciativa destacada ha sido la señal educativa transmitida por los canales de televisión de señal abierta y de cable, la cual contiene material educativo, en su mayoría generado a partir de fondos concursables del Consejo Nacional de Televisión (CNTV). Según una encuesta divulgada por esta misma institución, "un $86 \%$ de consultados manifiesta que canal TV Educa Chile debería continuar tras la contingencia sanitaria" (CNTV, 2020).

\section{Agencia docente y brechas sociales}

La emergencia sanitaria golpeó el sistema educativo con tal impacto que en pocas semanas se consiguieron avances en asuntos retrasados por años: crear y adaptar planes de estudio, contenidos y materiales para los nuevos entornos virtuales, ajustar metodologías y crear mecanismos de seguimiento y evaluación que permitan a los 160 millones de estudiantes latinoamericanos continuar con el derecho educativo. No obstante, "la acción pedagógica y las nuevas demandas encuentran al personal docente con una formación y una disponibilidad de recursos que tienden a ser insuficientes para los retos que supone adecuar la oferta y los formatos pedagógicos a estudiantes en entornos desfavorecidos" (CEPAL-Unesco, 2020, p. 10).

En efecto, el contexto ha creado un conjunto de nuevas responsabilidades que exceden la cuestión pedagógica, y con ello una consecuente sobrecarga laboral. Un estudio mostró que el 63 \% de docentes chilenos están trabajando más o mucho más que antes y en condiciones menos favorables (Elige Educar, 2020). La falta de recursos y capacidades instaladas, así como el recorte salarial y el aumento de tareas domésticas han generado de este modo un clima de mucha incertidumbre.

Asimismo, esta situación afecta en mayor medida a las mujeres. En la región latinoamericana la mayoría de docentes corresponde a este género: 
a nivel preescolar representan el $95.5 \%$, en primaria el $78.2 \%$ y en secundaria el 57.8 \%. Según la CEPAL y Unesco (2020):

Antes de la pandemia, las mujeres docentes debían enfrentar jornadas laborales dobles, que incluían no solo su trabajo en el aula, sino también las labores docentes fuera de ella (tareas administrativas, planificación y preparación de clases, entre otras), así como el trabajo doméstico y de cuidado no remunerado. (p. 10)

En esa línea, otro estudio del BID señala que:

Las madres tienen niveles particularmente bajos de bienestar, lo que podría ser un reflejo de que están asumiendo la mayor parte del apoyo educativo a distancia en el hogar (67\%) en comparación con los padres (26\%). En la mayoría de las familias, las mujeres también están a cargo de las comunicaciones con la escuela de sus hijos (71\%) en comparación con los hombres (solo el 16 \%). (Hernández-Agramonte et al., 2020)

Finalmente, sabemos que la educación puede ser un mecanismo de movilidad social para los estratos socioeconómicos más bajos o un vehículo de reproducción de las desigualdades sociales (Bourdieu \& Paterson, 2009; Hout \& DiPrete, 2006). Los resultados de la prueba PISA 2012 afirman lo segundo para Latinoamérica, una de las regiones con mayor desigualdad del mundo. La principal variable que marca la diferencia entre las competencias y aprendizajes de los estudiantes latinoamericanos es el nivel socioeconómico. La brecha de resultados por quintiles socioeconómicos es el doble que la de los países desarrollados (PNUD, 2017). Es en esta medida que nos interesa explorar, por un lado, las brechas identificadas en estudios previos $y$, por el otro, las condiciones materiales de los profesores y estudiantes como elementos que afectan sus prácticas e iniciativas docentes.

\section{Las percepciones docentes sobre sus capacidades TIC}

Desde hace un par de décadas existe evidencia empírica sobre los problemas y retos para la integración de TIC en la educación. Como señalan Cabero et al. (2020), en un ejercicio docente significativo y motivador confluyen diversos factores, "que van desde el apoyo institucional para la producción de objetos de aprendizaje para facilitar la incorporación de las TIC en la enseñanza, el grado de manejo instrumental que el profesorado tenga de las herramientas, sus competencias pedagógicas [...], y su adaptación a los contenidos curriculares" (p. 325). Ertmer (1999; 2005) dividió 
los factores entre aquellos llamados de primer orden, externos al docente, como el acceso material a dispositivos, los recursos técnicos y la capacitación con que se cuente, y los de segundo orden, vinculados con las creencias del propio docente sobre sus capacidades para interactuar con TIC, así como con las políticas y culturas escolares.

En el Perú, las autopercepciones han sido útiles para evaluar las competencias mediáticas en los docentes en formación y en ejercicio. La falta de entrenamiento específico y el limitado acceso a tecnologías en las escuelas fueron vistas por los futuros docentes como barreras importantes para desarrollar confianza en su actuación con las TIC (Mateus \& HernándezBreña, 2019), mientras que, para los docentes activos, la confianza en sí mismos aumenta con los años de formación y la experiencia acumulada (Gonzales-Miñán et al., 2020). En Chile, las competencias mediáticas autopercibidas por los docentes se reducen al uso instrumental de la tecnología, siguiendo las tendencias internacionales de la formación docente en medios (Andrada, 2015). En este contexto, las habilidades tecnológicas de los futuros docentes de nivel parvulario y básico, según la prueba INICIA TIC, son aceptables (Canales \& Hain, 2017). Mientras tanto, un estudio de caso mostró que los principales problemas docentes con las tecnologías se dan en las dimensiones pedagógicas y de gestión (Ascencio et al., 2016). En ese contexto, los docentes chilenos poseen altas expectativas acerca de los efectos positivos del uso de las TIC, tanto para enseñar como para aprender, aumentar el interés, adaptarse a las demandas de la sociedad actual o para conseguir reconocimiento. La tecnología es vista como un estímulo, por lo que prevalece un enfoque conductista que ve la motivación como algo externo al docente (Rioseco \& Roig, 2015).

Por otro lado, las percepciones docentes no solo se ven afectadas por sus actitudes o accesos materiales a la tecnología, sino también por el discurso sobre su rol e importancia social. Un estudio peruano aplicó una escala de autopercepción para medir las actitudes de los docentes y halló que existe un alto nivel de vocación y compromiso; no obstante, las bajas remuneraciones que perciben son un escollo relevante en la valoración de su profesión (Cuenca \& Carrillo, 2017).

En ese orden de ideas, las barreras subjetivas que construyen los docentes resultan importantes para explicar e incluso predecir el desempeño de una determinada experiencia (Mueller \& Wood, 2012; Sang et al., 2010). Sin embargo, "no todas las creencias y opiniones personales se construyen sobre variables estables y predecibles, muchas son arbitrarias y provienen de influenciadores exógenos como la idiosincrasia, la ideología o la experiencia" (Mateus, 2016, p. 174). No es suficiente considerar solo creencias o actitudes genéricas de los docentes sobre las tecnologías, sino también 
percepciones más específicas sobre lo que ellos consideran eficaz respecto al uso de estas, así como el vínculo que tienen con la naturaleza del conocimiento y del aprendizaje (Kima et al., 2013). Existe evidencia de que muchos docentes eligen y deciden el uso de ciertas tecnologías en función a su propia ontología (Tondeur et al., 2016).

"Los estudiantes perciben con claridad cuando sus docentes no se encuentran preparados para desarrollar su actividad profesional en contextos virtuales, lo que los lleva a desentenderse y despreocuparse por la formación" (Cabero et al., 2020, p. 338). Esto obliga a las instituciones a establecer medidas de contención y ayuda para sus docentes. No obstante, es importante apuntar que el abordaje no debe centrarse en el aspecto instrumental de los medios tecnológicos, es decir, en la enseñanza técnica de los dispositivos, sin reparar en su agencia cultural. La mediación tecnológica no se limita a la manipulación de hardware o software, sino a la comprensión profunda de las nuevas sensibilidades, prácticas y literacidades que generan en la interacción (Couldry \& Hepp, 2017; Martín-Barbero, 2009).

\section{Método y materiales}

\section{Enfoque metodológico}

Este trabajo se interesa en explorar las percepciones docentes sobre los cambios en sus prácticas y condiciones, respondiendo a la convocatoria internacional del proyecto EduCovid19. El enfoque cuantitativo de este trabajo es de alcance descriptivo, en tanto que busca dar cuenta de un fenómeno global, revelando características y rasgos importantes a partir de estudios comparativos. La finalidad fue analizar los cambios generados por la cancelación de las clases presenciales en casi todo el mundo y el obligado paso a la virtualidad, revelando condiciones de acceso y apropiación tecnológica asimétricas en los distintos países, y enumerando los retos inmediatos que esto supone para la comunidad educativa.

\section{Instrumento}

Para cumplir con el objetivo del estudio, un equipo de expertos del proyecto internacional diseñó un cuestionario que fue aplicado en diez países (Brasil, Chile, China, Chipre, Dinamarca, Colombia, México, Nigeria, Reino Unido y Uruguay). En este artículo damos cuenta de quince ítems de dicho cuestionario, organizados en torno a tres ejes temáticos: condiciones materiales de los docentes, competencias y acceso a TIC de estudiantes, y competencias y aprendizajes docentes (tabla 1). Asimismo, para el análisis descriptivo y por clústeres se tomaron en consideración las variables demográficas y educativas descritas en la tabla 2. 
Tabla 1.

Descripción del cuestionario

\begin{tabular}{|c|c|c|}
\hline Eje temático & Pregunta & $\begin{array}{l}\text { Tipo de } \\
\text { pregunta }\end{array}$ \\
\hline \multirow{5}{*}{$\begin{array}{l}\text { Condiciones } \\
\text { materiales } \\
\text { docentes } \\
\text { (5 ítems) }\end{array}$} & ¿Con cuáles de los siguientes dispositivos cuenta? & Opción múltiple \\
\hline & $\begin{array}{l}\text { ¿Cuál de las siguientes opciones refleja mejor su nivel de } \\
\text { conexión a internet en su hogar? }\end{array}$ & Nominal \\
\hline & $\begin{array}{l}\text { ¿Qué tan de acuerdo está con la siguiente afirmación?: } \\
\text { Cuento con un espacio en mi hogar adecuado para realizar mis } \\
\text { actividades de docencia en línea. }\end{array}$ & Likert \\
\hline & $\begin{array}{l}\text { ¿Qué tan de acuerdo está con la siguiente afirmación?: } \\
\text { Tengo acceso a las tecnologías que necesito usar para mis clases. }\end{array}$ & Likert \\
\hline & $\begin{array}{l}\text { ¿Qué tan de acuerdo está con la siguiente afirmación?: } \\
\text { Las personas que viven conmigo me apoyan para que pueda } \\
\text { realizar los cambios que requiero en mis clases. }\end{array}$ & Likert \\
\hline \multirow{3}{*}{$\begin{array}{l}\text { Competencias } \\
\text { y acceso a TIC } \\
\text { en estudiantes } \\
\text { ( } 3 \text { ítems) }\end{array}$} & $\begin{array}{l}\text { ¿Qué tan de acuerdo está con la siguiente afirmación?: } \\
\text { Mis estudiantes cuentan con las habilidades necesarias para } \\
\text { estudiar en línea. }\end{array}$ & Likert \\
\hline & $\begin{array}{l}\text { ¿Qué tan de acuerdo está con la siguiente afirmación?: } \\
\text { Mis estudiantes cuentan con los dispositivos necesarios } \\
\text { (computadora o tableta) para estudiar en línea. }\end{array}$ & Likert \\
\hline & $\begin{array}{l}\text { ¿Qué tan de acuerdo está con la siguiente afirmación?: } \\
\text { Mis estudiantes cuentan con el acceso necesario a internet } \\
\text { para estudiar en línea. }\end{array}$ & Likert \\
\hline \multirow{7}{*}{$\begin{array}{l}\text { Competencias } \\
\text { y aprendizajes } \\
\text { docentes } \\
\text { (7 ítems) }\end{array}$} & $\begin{array}{l}\text { ¿Cómo percibe que es su competencia en el uso } \\
\text { de tecnologías educativas? }\end{array}$ & Likert \\
\hline & $\begin{array}{l}\text { ¿Qué tan preparado(a) se ha sentido para realizar los cambios } \\
\text { requeridos en sus clases? }\end{array}$ & Likert \\
\hline & $\begin{array}{l}\text { A partir de la pandemia del covid-19, ¿ha aprendido nuevas } \\
\text { formas de impartir sus clases? }\end{array}$ & Opción múltiple \\
\hline & $\begin{array}{l}\text { ¿Qué competencias ha buscado desarrollar para realizar } \\
\text { los cambios requeridos en sus cursos a partir de la pandemia } \\
\text { del covid-19? }\end{array}$ & Opción múltiple \\
\hline & $\begin{array}{l}\text { ¿Qué tan de acuerdo está con la siguiente afirmación?: } \\
\text { Conozco lo suficiente sobre enfoques pedagógicos apropiados } \\
\text { para apoyar el aprendizaje en línea. }\end{array}$ & Likert \\
\hline & $\begin{array}{l}\text { ¿Qué tan de acuerdo está con la siguiente afirmación?: } \\
\text { Estoy dispuesto(a) a experimentar para modificar } \\
\text { mis prácticas docentes. }\end{array}$ & Likert \\
\hline & Tengo confianza en mi capacidad para enseñar en línea. & Likert \\
\hline
\end{tabular}

Fuente: Elaboración propia

El coeficiente del alfa de Cronbach arrojó un resultado de 0.887 , lo que confirma la fiabilidad del instrumento. Para hallar diferencias significativas se hicieron comparaciones de proporciones por columnas mediante el análisis de varianza, aplicando el método de Bonferroni para ajustar los valores de significación. 


\section{Muestra}

El cuestionario fue aplicado a todos los docentes que quisieron participar de forma voluntaria y que estaban dando clases, es decir que se realizó una muestra por conveniencia. Participaron 541 profesores, luego de excluir los casos de respuestas incompletas o de personas que no residían en ninguno de los dos países analizados. La muestra final de participantes se distribuyó de la siguiente forma:

Tabla 2.

Variables y muestra

\begin{tabular}{|c|c|}
\hline Variables & Muestra $(\mathrm{N}=541)$ \\
\hline \multicolumn{2}{|c|}{ País } \\
\hline Perú & $46 \%$ \\
\hline Chile & $54 \%$ \\
\hline \multicolumn{2}{|c|}{ Género } \\
\hline Femenino & $63 \%$ \\
\hline Masculino & $35 \%$ \\
\hline Prefiero no decirlo & $2 \%$ \\
\hline \multicolumn{2}{|c|}{ Edad } \\
\hline Hasta 35 años & $26 \%$ \\
\hline 36 a 49 años & $46 \%$ \\
\hline 50 a más años & $28 \%$ \\
\hline \multicolumn{2}{|c|}{ Experiencia } \\
\hline Menos de 10 años & $46 \%$ \\
\hline 10 años a más & $54 \%$ \\
\hline \multicolumn{2}{|c|}{ Entorno educativo } \\
\hline Preescolar/Inicial & $11 \%$ \\
\hline Primaria & $21 \%$ \\
\hline Secundaria & $33 \%$ \\
\hline Superior & $32 \%$ \\
\hline Otros & $4 \%$ \\
\hline \multicolumn{2}{|c|}{ Tipo de institución } \\
\hline Público & $35 \%$ \\
\hline Privado & $56 \%$ \\
\hline Otros & $9 \%$ \\
\hline \multicolumn{2}{|l|}{ Ámbito } \\
\hline Capital & $57 \%$ \\
\hline Regiones (provincias) & $43 \%$ \\
\hline \multicolumn{2}{|c|}{ Tipo de localidad } \\
\hline Urbana & $88 \%$ \\
\hline Sub-urbana & $4 \%$ \\
\hline Rural & $7 \%$ \\
\hline \multicolumn{2}{|c|}{ Nivel educativo } \\
\hline Secundaria o Bachillerato & $0 \%$ \\
\hline Licenciatura o Técnica & $51 \%$ \\
\hline Maestría & $39 \%$ \\
\hline Doctorado & $10 \%$ \\
\hline
\end{tabular}

Fuente: Elaboración propia 


\section{Procedimiento y análisis}

El cuestionario fue aplicado por medio de un formulario en línea difundido a través de redes docentes en ambos países. La participación fue voluntaria e informada. El periodo de aplicación fue del 25 de mayo al 30 de junio de 2020. Los resultados cuantitativos fueron procesados en el programa IBM SPSS Statistics $24{ }^{\circledR}$.

\section{Resultados}

\section{Condiciones materiales de los docentes}

En cuanto al acceso a tecnología, la mayoría de los docentes participantes en el estudio señaló que tiene una computadora portátil (90.9\%), seguido de teléfonos inteligentes (80.4\%) o tabletas (31.8\%). Los docentes peruanos tienen más computadoras de escritorio, y sus pares chilenos, un mayor número de ordenadores portátiles. A nivel etario, los docentes menores de 35 años tienen más smartphones que los mayores de 50, mientras que los docentes de educación superior cuentan con muchos más de estos aparatos que los de secundaria.

Son igualmente significativas las diferencias entre los docentes de educación privada, con más acceso a tabletas que los de instituciones públicas. Igualmente, los docentes que trabajan en las capitales tienen más computadoras de escritorio que los de las regiones y los que lo hacen en zonas urbanas, más teléfonos inteligentes y tabletas que sus colegas de zonas rurales.

Con relación a la conectividad a internet, la mayoría cuenta con un plan ilimitado (63\%). Sin embargo, hay diferencias significativas entre niveles: los docentes de los niveles preescolar, primaria y secundaria usan más los datos de sus teléfonos móviles para conectarse que sus pares de enseñanza superior, quienes acceden en mayor medida a planes ilimitados, confirmando mejores posibilidades de conexión.

Al igual que en el punto anterior, destaca la diferencia entre los docentes de instituciones privadas y públicas; mientras los primeros acceden a internet mediante planes ilimitados, los segundos dependen mucho más de sus datos móviles o de planes limitados. Igualmente, los docentes de las ciudades capitales, Santiago y Lima, tienen mayor acceso ilimitado que los de regiones, tal como los que laboran en zonas urbanas en comparación con las rurales (gráfico 1).

Poco más de la mitad de los docentes (54.2\%) señaló que contaba con un espacio adecuado en su hogar para desarrollar sus clases. En este punto, las mujeres acusan tener menos espacios que sus pares hombres. Asimismo, destacan diferencias significativas a favor de los docentes mayores de 50 años respecto a los menores de 35, y de los profesores de 
educación superior frente a sus pares de educación primaria. En general, los docentes del nivel terciario tienen mejores condiciones en el hogar que sus pares del nivel primario y secundario (gráfico 2).

Gráfico 1.

Diferencias de conectividad a internet por variables

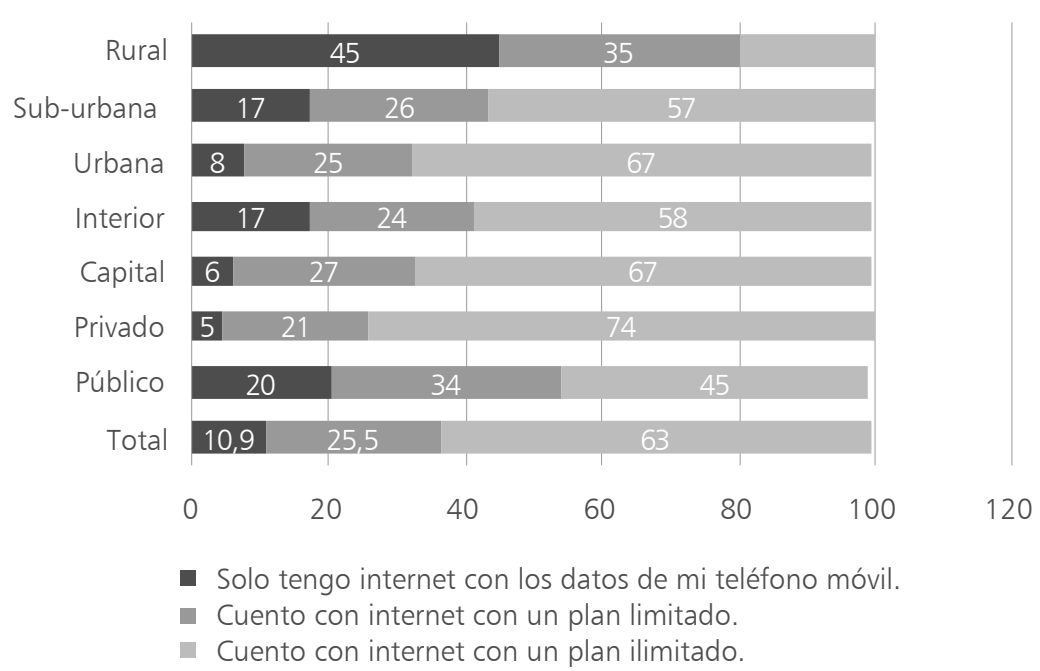

Gráfico 2.

Condiciones hogareñas de los docentes para realizar sus clases

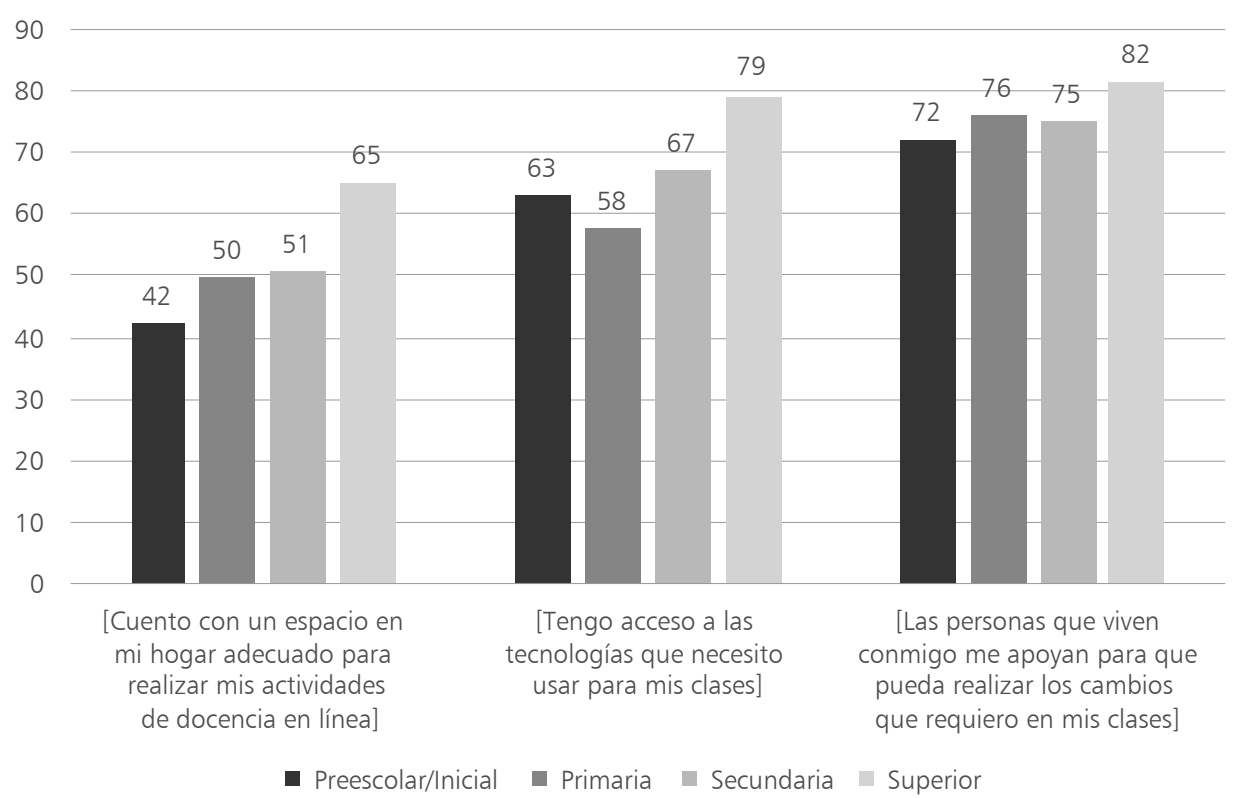


Para dar las clases, la mayoría de participantes en el estudio (68.4\%) manifestó que cuenta con la tecnología necesaria. Si bien la mayoría de docentes respondió de forma positiva, el número de docentes mujeres que señaló no contar con tecnologías necesarias para sus clases duplica al de los hombres. Otra diferencia muy marcada es la de los docentes de instituciones privadas (75.4\%) frente a los que trabajan en instituciones públicas (58.9\%). Igualmente, las respuestas negativas del sector rural y suburbano duplican al urbano. En el caso peruano también es marcada la diferencia entre la capital (74.7 \%) y las regiones (56.5\%).

Finalmente, sobre el apoyo que los docentes sentían de las personas con quienes comparten el hogar, la diferencia más marcada se aprecia en un mayor apoyo a los docentes de instituciones privadas que de las públicas.

\section{Condiciones y capacidades de los estudiantes}

En cuanto a las habilidades necesarias para estudiar en línea, son más los docentes que señalan que sus estudiantes no las tienen (43.8\%) que los que sí (37.3 \%). Las diferencias significativas apuntan a variables generacionales: los docentes mayores de 50 años confían más en la preparación de sus estudiantes que sus colegas menores de 35; los que tienen menos de 10 años en la docencia son más críticos con las capacidades de sus estudiantes que sus colegas con más de 10; también, los profesores de educación superior valoran mejor las capacidades de sus estudiantes que sus colegas de la educación primaria.

Otras diferencias significativas son las de los docentes de instituciones privadas, quienes aprecian mejores habilidades de sus discentes que los de instituciones públicas, lo mismo que los que laboran en las capitales, a diferencia de sus pares que trabajan en regiones (lo que resulta más marcado en el caso peruano). Asimismo, los docentes que tienen un doctorado ven mejores capacidades en sus alumnos que los que tienen licenciatura.

Cuando indagamos sobre el acceso de sus estudiantes a dispositivos necesarios (computadoras o tabletas), la mayoría de docentes señala que esto no se cumple (48.8\%). Como era previsible por las brechas estructurales, la diferencia más significativa la encontramos entre los docentes de instituciones públicas, quienes acusaron mayores carencias materiales de sus estudiantes (75.5\%) que sus pares de instituciones privadas (29.6\%). Respecto del nivel educativo, la visión es significativamente más negativa de parte de los docentes de preescolar, primaria y secundaria que de sus pares de educación superior.

A nivel territorial, la diferencia entre docentes que laboran en las capitales y las regiones es algo más favorable en el caso de los primeros a 
nivel global, aunque hay diferencia entre los países. En el caso peruano, los docentes regionales duplican en respuestas negativas a sus colegas de Lima; mientras que en Chile hay más respuestas negativas en Santiago que en las regiones. En cuestión generacional y de experiencia, los docentes menores de 35 años y los que tienen menos de 10 años como docentes fueron más negativos que sus pares mayores sobre el acceso a TIC de sus estudiantes.

Finalmente, en relación con la conectividad, de manera coincidente con la pregunta anterior, la mayoría de docentes (49.7 \%) señala que sus estudiantes no cuentan con ella de forma estable, mientras que menos de un tercio señalan que sí (31.8\%). Encontramos aquí diferencias significativas en cuatro de las variables analizadas.

La brecha más marcada nuevamente se da entre las instituciones públicas (gráfico 3), donde la gran mayoría de docentes percibe que los estudiantes no tienen un buen acceso a internet (72.9\%), más que duplicando la percepción que existe entre los privados (33.2 \%). Los profesores más jóvenes tienen una peor percepción de la calidad del internet de sus estudiantes. Similar situación ocurre con los docentes con menos experiencia que los que llevan más de 10 años ejerciendo la profesión. Al igual que en el caso del acceso a dispositivos, a nivel general las brechas no son sustantivas (gráfico 3), aunque existen diferencias territoriales entre los países. En las regiones rurales de Perú existe una peor percepción del acceso a internet por parte de sus estudiantes, mientras en Chile esta peor percepción se da en la capital.

Gráfico 3.

Falta de acceso a internet de estudiantes por tipo de institución y ámbito

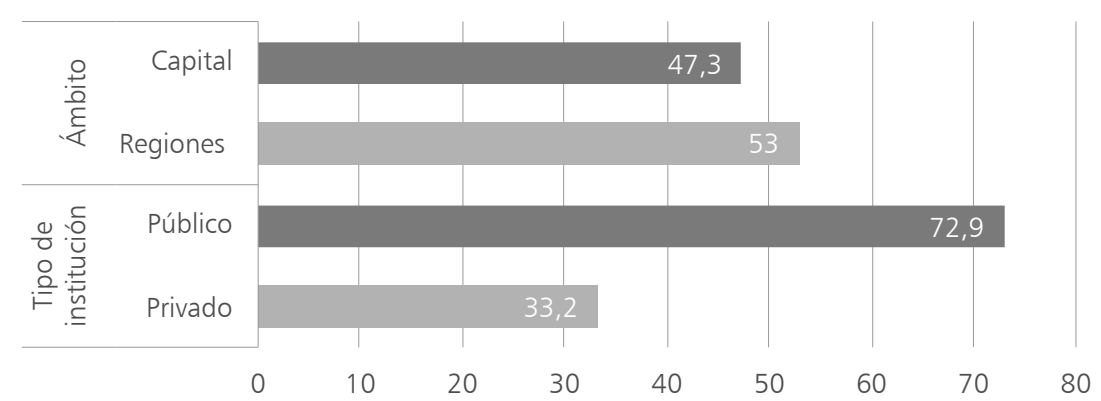

Fuente: Elaboración propia

\section{Competencias y aprendizajes docentes}

La mayoría de docentes participantes (70.2 \%) tiene una percepción positiva de su competencia en el uso de tecnologías educativas. Los docentes 
identificados con el género masculino lo perciben en mayor magnitud que sus pares del género femenino (gráfico 4). Asimismo, aquellos docentes con estudios de posgrado (máster y doctorado) tienen una autopercepción superior a sus pares con grado de licenciatura.

Respecto a qué tan preparado se ha sentido para adaptar sus clases, la respuesta mayoritaria (61.9\%) vuelve a ser favorable. Aquí se repite la diferencia significativa en favor de los docentes del género masculino (gráfico 4).

Gráfico 4.

Percepción de competencias y grado de preparación docente por variable género

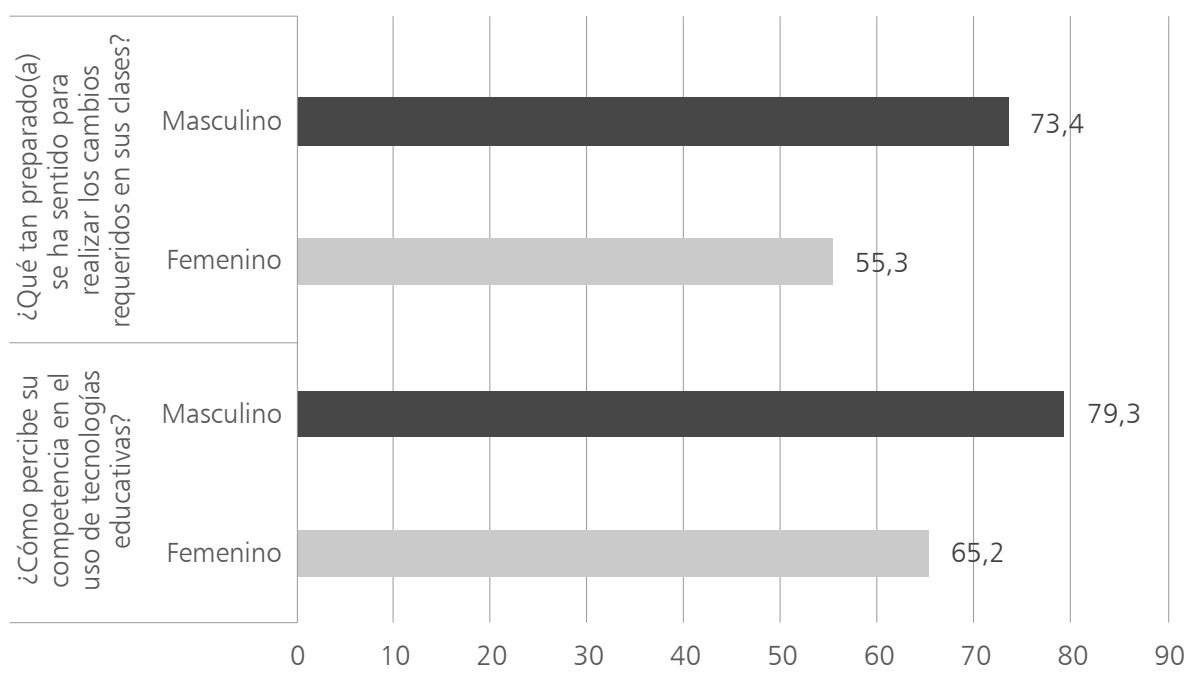

Fuente: Elaboración propia

Los docentes manifestaron en su gran mayoría haber aprendido nuevas cosas para adaptar sus clases a la emergencia y haberse preparado por cuenta propia y viendo recursos en línea, en primer lugar, y, en segundo, asistiendo a capacitaciones gratuitas (talleres, MOOC o diplomados).

Los profesores que más se prepararon por cuenta propia fueron significativamente los jóvenes y los de mediana edad (36 a 49 años), los que tienen menos experiencia, los de primaria y secundaria, los de instituciones públicas y los que tienen el grado de licenciatura. En cambio, en cuanto a participación en capacitaciones gratuitas destacan las mujeres, los profesores universitarios, los de instituciones privadas y quienes tienen estudios de posgrado (máster y doctorado).

Cuando exploramos qué competencias habían buscado desarrollar para poder adaptar sus cursos al escenario de educación remota destacaron, en primer lugar, las capacidades tecnológicas (85\%) y, en segundo, las pedagógicas (65\%). Las capacidades tecnológicas aluden al uso de 
herramientas digitales específicas, como algún programa o dispositivo; mientras que las pedagógicas se vinculan con el conocimiento de estrategias didácticas para enseñar en línea.

Las diferencias significativas se dan principalmente en el ámbito de las competencias pedagógicas. Dentro de los encuestados, fueron los docentes peruanos quienes buscaron más competencias pedagógicas que sus pares chilenos, los de mediana edad (36 a 49 años) más que los mayores y los de primaria y superior más que los de preescolar (gráfico 5). La única excepción fueron los profesores que llevan más de 10 años, quienes buscaron más competencias tecnológicas que sus pares con menor experiencia.

Gráfico 5.

Demanda de competencias pedagógicas por país, edad y entorno educativo

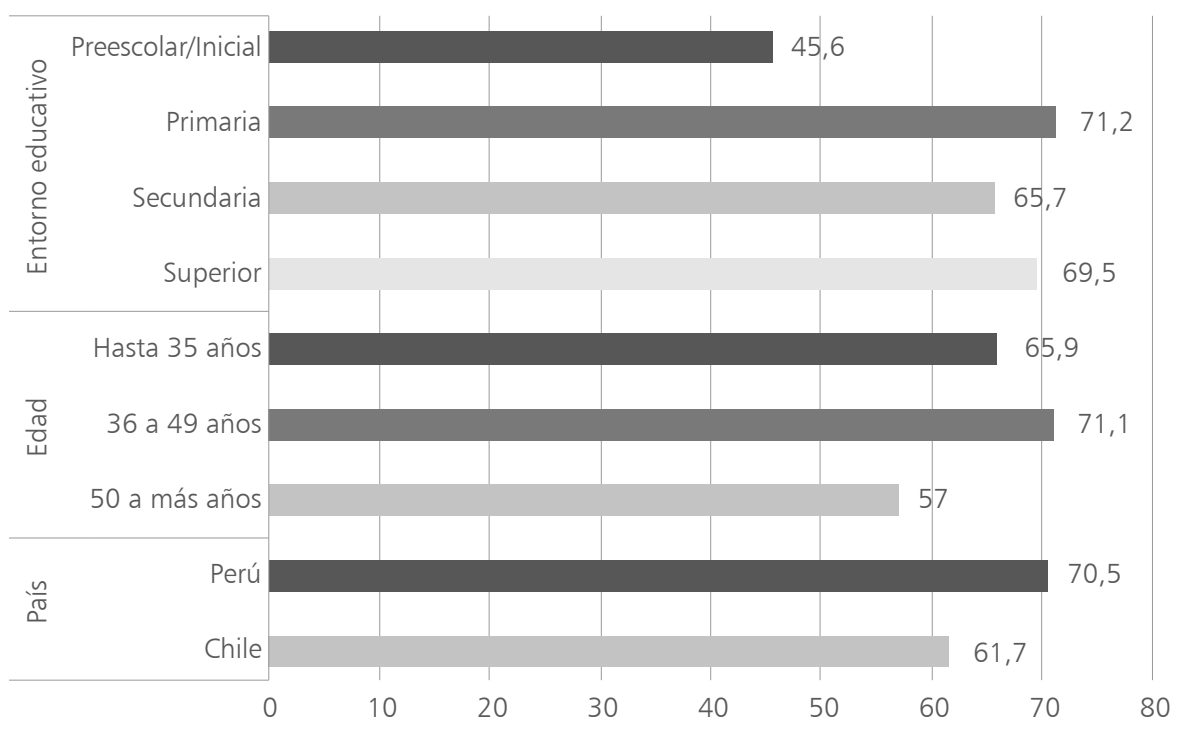

Fuente: Elaboración propia

Al preguntarles si conocían lo suficiente sobre enfoques pedagógicos apropiados para apoyar el aprendizaje virtual, la mayoría de los profesores afirmó que sí (45.3\%), mientras una cuarta parte de los encuestados respondió negativamente. Los docentes peruanos fueron más positivos que sus pares chilenos, y los profesores de género masculino también lo fueron respecto de sus pares mujeres (gráfico 6). En materia de edades, los profesores con más de 50 años se sienten más confiados que los menores de 35 , lo que resulta consistente con la tendencia en aquellos docentes de mayor experiencia (más de 10 años), respecto de los otros. También, la confianza en sus conocimientos pedagógicos sube con relación a los grados académicos que ostentan (mayor en los que tienen doctorados y másteres que los que tienen licenciatura). 
La situación llevó a muchos a experimentar sobre la marcha, haciendo un ejercicio de ensayo y error. En ese sentido, la gran mayoría (86.1 \%) manifiesta su disposición a probar nuevas cosas. La diferencia es significativa a favor de los hombres y, en el caso territorial, los docentes de áreas urbanas duplican en porcentaje a los de instituciones rurales. De la misma forma, la seguridad de querer experimentar aumenta conforme el nivel educativo, siendo significativamente mayor en los de educación superior y secundaria que en los de nivel preescolar.

Respecto a la confianza que los docentes tienen en su capacidad de enseñar en línea, la gran mayoría se siente confiado o muy confiado (83.4\%). Dentro de este campo, las diferencias significativas se dan en cuatro variables. Los profesores peruanos se sienten más confiados que los chilenos, los hombres más que las mujeres (gráfico 6), los de educación superior y secundaria más que los de educación preescolar y los que tienen estudios de doctorado más que los que tienen licenciatura.

Gráfico 6.

Grados de autoconfianza de docentes por variable género

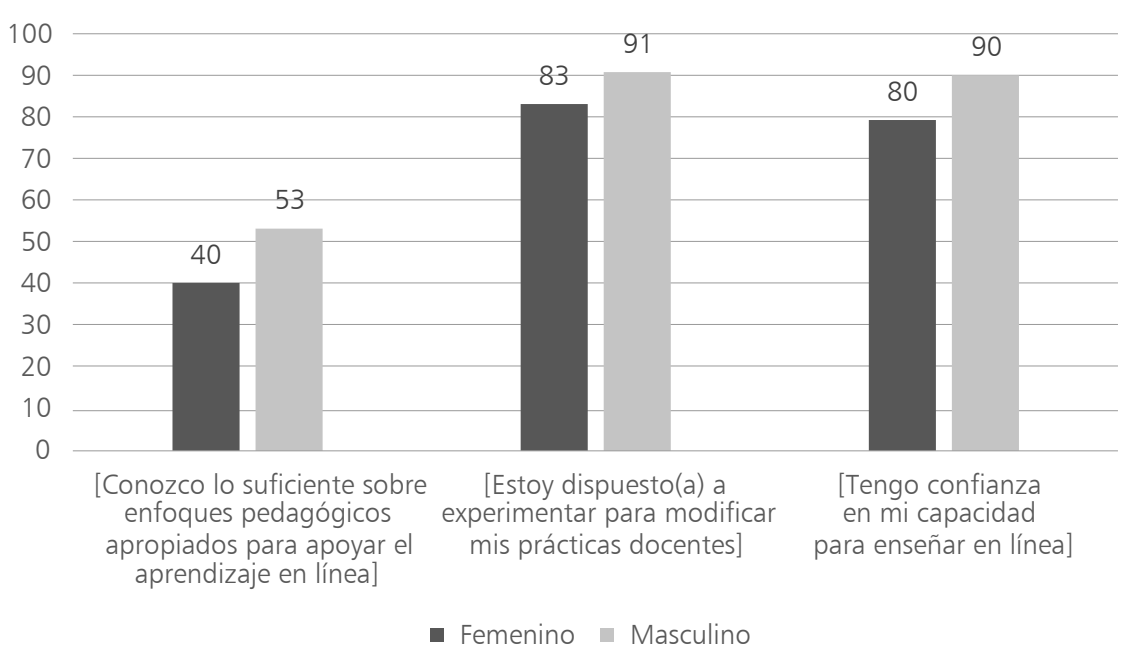

Fuente: Elaboración propia

\section{Discusión de resultados}

Los resultados de este estudio, elaborado en el contexto de la emergencia sanitaria causada por el covid-19, nos permiten confirmar el impacto de las brechas estructurales, sociales y demográficas en la forma como los docentes perciben sus competencias. Del mismo modo, se deduce cómo estas diferencias también afectan significativamente las competencias de sus estudiantes. A pesar de que la autopercepción tiene un 
alto componente subjetivo, nos permite enunciar los problemas y brechas desde las vivencias concretas de los docentes. En ese sentido, esta metodología resulta válida para reconocer escenarios complejos puestos en evidencia por esta pandemia.

Las diferencias entre los sectores urbano o rural, así como entre una institución pública o privada, afectan la percepción de los docentes sobre sus condiciones y prácticas. Adicionalmente, las variables de género y edad permiten reportar diferencias significativas que conviene atender. El perfil del docente chileno y peruano menos favorecido en este contexto tiene nombre de mujer, grado de licenciada, y trabaja en una escuela pública y rural de alguna región de estos países.

El análisis por clústeres nos permite concluir que existen algunas diferencias significativas que destacan sobre otras en cada dimensión. En primer término, las condiciones materiales resultan más importantes con relación al nivel educativo en que el docente se desenvuelve. Así, los docentes de los niveles preescolar o primaria tienen una peor autopercepción frente a sus pares de educación secundaria o superior. Esto podría atribuirse a que el tipo de recursos didácticos que demanda el trabajo con estudiantes más pequeños es más complejo por el tipo de métodos que aplican. En ese sentido, sería indicado atender la demanda de conocimiento de estrategias didácticas en línea para este sector específico. En este nivel también destacan las condiciones poco favorables que tienen las mujeres, los docentes de educación pública y del ámbito rural. Esto reclama invertir mayores recursos para igualar las oportunidades de estos grupos.

En la autopercepción de sus capacidades como docentes, destaca la diferencia en cuanto al género. Las docentes mujeres acusan tener menores capacidades que sus pares hombres. Esta percepción se relaciona con las peores condiciones materiales y, por lo tanto, menores recursos económicos disponibles para ejercer su labor. En correspondencia con lo anterior, las docentes cuentan con menor tiempo para perfeccionar sus capacidades. Es relevante la presión mayor sobre las mujeres, vinculada con una sobrecarga laboral, como afirman otros estudios (CEPAL-Unesco, 2020; Elige Educar, 2020; Hernández-Agramonte et al., 2020). Es urgente responder a estas disparidades desde el enfoque de género. En el ámbito educativo, se propone tomar en consideración especialmente esta situación de sobreexigencia a la que se ven sometidas las mujeres para evitar afectar sus condiciones socioemocionales de trabajo.

Igual que las docentes mujeres, los docentes con un menor grado académico se consideran menos confiados sobre sus competencias. Esto es explicado en la literatura por la limitada experiencia, que juega en contra de 
los juicios de valor que el docente establece sobre sí mismo (Ertmer, 1999; 2005). Es necesario afrontar esta barrera con políticas de reconocimiento de buenas prácticas docentes, sea a través de sus redes de colegas o de las propias instituciones. Este reconocimiento les permitiría acreditar y compartir los saberes que van construyendo por cuenta propia. Una alternativa complementaria es favorecer programas de perfeccionamiento que vayan promoviendo mayor confianza sobre sus capacidades, y no limitarlos a exámenes punitivos.

De forma correspondiente, en el eje de valoración de las capacidades de los estudiantes, resulta que los docentes con más edad y años de experiencia valoran mejor las capacidades tecnológicas de sus alumnos. Parece lógico que, con la edad, aumente de forma correlativa la distancia entre las posibilidades técnicas de estudiantes y docentes. Por eso, los docentes más jóvenes son más críticos de las competencias de sus estudiantes por su cercanía generacional. Más que igualar las capacidades, sugerimos valorar los saberes diferenciados de cada actor, promoviendo un diálogo intergeneracional empático y respetuoso. Asimismo, como marca la evidencia, el que los estudiantes parezcan más hábiles en la manipulación de los dispositivos, no les confiere una competencia digital crítica: existe una actitud y una finalidad formativa que no surge de forma espontánea y que es misión del propio docente promover (Ferrés et al., 2018; Raffaghelli, 2020).

Finalmente, es inútil pensar las consecuencias de esta crisis omitiendo su carácter político. En línea de lo planteado por Giroux et al. (2020):

[...] el modelo pedagógico basado en la idea de que la responsabilidad individual como única forma de abordar los problemas sociales y, en consecuencia, sin necesidad de abordar cuestiones de responsabilidad colectiva, nos ha hecho más débiles para enfrentar una catástrofe como la que estamos viviendo. (p. 5)

Los resultados confirman carencias y brechas estructurales presentes en ambos sistemas educativos antes de la pandemia. La circunstancia de emergencia las amplificó, obligando a los docentes a depender de infraestructuras y entornos mediáticos y digitales precarios y limitados. A partir de la evidencia podemos señalar que en ambos países logró constituirse un primer nivel de respuesta a partir de la provisión de contenidos pedagógicos en algunos medios, pero que en la práctica operó sobre todo la lógica del "salvase quien pueda y con lo que tenga". Esto no solo repercute en los estudiantes más vulnerables, sino también en los docentes, cuya condición se vio agravada. 


\section{Conclusión}

La educación tiende a reproducir las condiciones sociales de los estudiantes. Más que un lugar de ascenso social es un espacio donde se perpetúa la herencia del lugar de nacimiento (Bourdieu \& Paterson, 2009). La pandemia del covid-19 ha sido un triste recordatorio de lo anterior.

En los últimos treinta años los gobiernos latinoamericanos han intentado dar el salto digital con programas de formación en TIC, con resultados en gran medida negativos. En la mayoría de ellos, la acción tecnológica ha tenido prioridad sobre la pedagógica (Mateus et al., 2020). Más aún, se reducen los medios al uso de la tecnología y no se los percibe en sus múltiples dimensiones.

Asimismo, la virtualidad tiende a la idea errónea de que el sistema educativo es un sistema de transmisión que depende de la voluntad del estudiante de aprender y del docente de enseñar. No es verdad. Los estudiantes no deciden abandonar las escuelas ni los docentes evitan experimentar por falta de interés: son las condiciones desiguales las que los predisponen a hacerlo. Bajo esta lógica, nada sería peor que proponer políticas públicas unidimensionales que insistan en la visión instrumental de las tecnologías, es decir, que se limiten a la distribución de dispositivos y no preparen al docente para comprender el medio tecnológico como agente cultural generador de nuevas sensibilidades (Martín-Barbero, 2009). Por el contrario, los resultados invitan a promover proyectos que tengan como fin el empoderamiento de los docentes en su rol de mediadores con las tecnologías y que potencien actitudes colaborativas y solidarias, en ellos y entre sus instituciones y las comunidades en las que impactan.

Este estudio reafirma el alto impacto de las brechas socioeconómicas y de género, así como de las condiciones materiales, en el ejercicio profesional docente. Algunas limitaciones de este trabajo están asociadas a que los hallazgos son aproximaciones a la realidad y no buscan ser representativos, ya que las muestras fueron por conveniencia. Al respecto, a pesar de contar con más de 500 casos, existieron dificultades en la recolección de los datos, sobre todo de los sectores rurales, por las restricciones de conectividad. Asimismo, el tiempo de ejecución (mayo-junio de 2020) corresponde a lo que hoy podemos llamar un momento inicial de la pandemia. Sería importante realizar un estudio longitudinal que evalúe respuestas de la misma población un año después, por ejemplo. Finalmente, los hallazgos comunes entre Chile y Perú sugieren la posibilidad de estudiar nuevas comparaciones con otros sistemas educativos y la búsqueda conjunta de soluciones que luego puedan ser adaptadas a cada contexto. Aun sin mayor certeza sobre el final 
de este proceso histórico, podemos inferir que algunos de los cambios iniciados con las estrategias de educación remota trascenderán el escenario pandémico, sobre todo aquellos vinculados al desarrollo de capacidades mediáticas de los docentes, así como a la producción de contenidos didácticos a través de las TIC.

\section{Agradecimientos}

Los autores agradecen a Antonio Ruiz-Olorte por su trabajo en el procesamiento de datos y elaboración de cuadros estadísticos.

\section{Sobre los autores}

Julio-César Mateus es profesor asociado e investigador de la Facultad de Comunicación de la Universidad de Lima, Perú. Coordinador del grupo de investigación en Comunicación y Educación del Instituto de Investigación Científica. Miembro de la Red Interuniversitaria EuroAmericana de Investigación sobre competencias mediáticas para la ciudadanía (Alfamed) y de la Asociación Latinoamericana de Investigadores en Comunicación (ALAIC).

Pablo Andrada es profesor de la Facultad de Ciencias Sociales, Empresariales y Jurídicas de la Universidad de La Serena, de Chile. Miembro de la Asociación de Investigadores en Comunicación de Chile, Incom; de la Red Interuniversitaria EuroAmericana de Investigación sobre competencias mediáticas para la ciudadanía (Alfamed) y de la Asociación Latinoamericana de investigadores en Comunicación (ALAIC).

\section{Referencias}

Alayo Orbegozo, F. (28 de setiembre de 2020). Unos 174.000 estudiantes peruanos dejaron la universidad en lo que va del 2020. El Comercio. https:// elcomercio.pe/lima/sucesos/unos-174000-estudiantes-peruanos-dejaronla-universidad-en-lo-que-va-del-2020-noticia/

Andrada, P. (2015). Estado de la cuestión de la formación docente en educación en medios. Comunicación y Medios, (32), 83-99. https://doi.org/10.53 54/0719-1529.2015.36700

Arraigada-Toledo, P. (2020). Pandemia Covid-19: Educación a distancia. O las distancias en la educación. Revista Internacional de Educación para la Justicia Social, 9(3). https://revistas.uam.es/riejs/article/view/12396

Ascencio, P., Garay, M. \& Seguic, E. (2016). Formación Inicial Docente (FID) y Tecnologías de la Información y Comunicación (TIC) en la Universidad de Magallanes - Patagonia Chilena. Digital Education Review, 30, 135-146. https://dialnet.unirioja.es/servlet/articulo?codigo $=5772410$

Bourdieu, P. \& Paterson, J.-C. (2009). Los herederos: los estudiantes y la cultura. Siglo XXI. 
Busso, M. \& Messina, J. (eds.). (2020). La crisis de la desigualdad: América Latina y el Caribe en la encrucijada. Banco Interamericano de Desarrollo. http:// dx.doi.org/10.18235/0002629

Cabero, J. C., Del Prete, A. \& Arancibia, M. L. (2020). Modelo para determinar acciones de calidad en la formación virtual. Digital Education Review, 37, 323-342. https://doi.org/10.1344/der.2020.37.323-342

Cadem. (2020). Plaza Pública Nº 348- 14 de septiembre [Encuesta]. Cadem.

Canales, R. \& Hain, A. (2017). Política de informática educativa en Chile: uso, apropiación y desafíos a nivel investigativo. En R. Cabello \& A. López (Eds.), Contribuciones al estudio de procesos de apropiación de tecnologías (pp. 131-144). Gato Gris.

CEPAL-Unesco. (2020). La educación en tiempos de la pandemia de COVID-19 [Informe]. https://unesdoc.unesco.org/ark:/48223/pf0000374075?locale=es

CNTV - Consejo Nacional de Televisión. (2020). Un 86 \% de consultados manifiesta que Canal TV Educa Chile debería continuar tras la contingencia sanitaria. https://www.cntv.cl/2020/06/02/86-de-consultados-manifiesta-que-canaltv-educa-chile-deberia-continuar-tras-la-contingencia-sanitaria/

Couldry, N. \& Hepp, A. (2017). The Mediated Construction of Reality. Polity Press.

Cuenca, R. \& Carrillo, S. (2017). Una mirada a la profesión docente en el Perú: futuros docentes, docentes en servicio y formadores de docentes. Unesco/Ministerio de Educación. http://repositorio.minedu.gob.pe/hand le/20.500.12799/5711

Cuenca, R. \& Urrutia, C. E. (2019). Explorando las brechas de desigualdad educativa en el Perú. Revista Mexicana de Investigación Educativa, 24(81), 431461. http://ref.scielo.org/646mr4

Cueto, S., Felipe, C. \& León, J. (2020). Predictores de la deserción escolar en el Perú [Análisis \& Propuestas, 52]. GRADE, Niños del Milenio. http://www. grade.org.pe/wp-content/uploads/GRADEap52.pdf

Diario de Concepción (17 de septiembre 2020). El peligro de la deserción escolar. https://www.diarioconcepcion.cl/editorial/2020/09/17/el-peligro-de-ladesercion-escolar.html

El Comercio (24 de agosto de 2020). Educación ante la pandemia: Conectividad en regiones y acceso a equipos limitan programa "Aprendo en casa". https://elcomercio.pe/economia/peru/educacion-ante-la-pandemia-conec tividad-en-regiones-y-acceso-a-equipos-limitan-programa-aprendo-encasa-noticia/

Elige Educar. (2020). Situación de docentes y educadores en contexto de pandemia: reporte de resultados. https://eligeeducar.cl/wp-content/ uploads/2020/05/Resultados_EncuestaEEcovid_SitioWeb_mi.pdf

Ertmer, P. (1999). Addressing first- and second-order barriers to change: Strategies for technology integration. Educational Technology Research and Development, 47(4), 47-61. https://doi.org/10.1007/BF02299597

Ertmer, P. (2005). Teacher pedagogical beliefs: The final frontier in our quest for technology integration? Educational Technology Research and Development, 53, 25-39. https://doi.org/10.1007/BF02504683 
Ferrés, J., Masanet, M. J. \& Mateus, J. C. (2018). Three paradoxes in the approach to educational technology in the education studies of the Spanish universities. International Journal of Educational Technology in Higher Education, 15(1), 1-15. http://dx.doi.org/10.1186/s41239-018-0097-y

Figallo, F., González, M. T. \& Diestra, V. (2020). Perú: Educación superior en el contexto de la pandemia por el COVID-19. Revista de Educación Superior en América Latina, (8), 20-28. https://rcientificas.uninorte.edu.co/index.php/ esal/article/view/13404

Giroux, H., Rivera-Vargas, P. \& Passeron, E. (2020). Pedagogía pandémica. Reproducción funcional o educación antihegemónica. Revista Internacional de Educación para la Justicia Social, 9(3). https://revistas.uam.es/riejs/article/ view/12199

Gonzales-Miñán, M. del C., Turpo-Gebera, O. \& Suárez-Guerrero, C. (2020). La autopercepción de competencia mediática y su relación con las variables sociodemográficas del profesorado de tres instituciones educativas ubicadas en Lima. Aula Abierta, 49(2), 103-11. https://doi.org/10.17811/rifie. 49.2.2020.103-11

Hernández-Agramonte, J., Lisboa-Vásquez, C. P., Méndez, C., Namen, O., Näslund-Hadley, E. \& Velarde, L. (2020). 8,000 familias en Perú nos cuentan sobre el costo socioemocional de la pandemia COVID-19. Enfoque Educación. BID. https://blogs.iadb.org/educacion/es/costosocioemocionalcovid19/

Hout, M., \& DiPrete, T. A. (2006). What we have learned: RC28's contributions to knowledge about social stratification. Research in Social Stratification and Mobility, 24 (1), 1-20. https://doi.org/10.1016/j.rssm.2005.10.001

Kima, C., Kima, M. K., Lee, C., Spector, J. M. \& DeMeester, K. (2013). Teacher beliefs and technology integration. Teaching and Teacher Education, (29), 76-85. http://dx.doi.org/10.1016/j.tate.2012.08.005

Martín-Barbero, J. (2009). Cuando la tecnología deja de ser una ayuda didáctica para convertirse en mediación cultural. Teoría de la Educación, 10(1), 19-31. https://doi.org/10.14201/eks.15549

Mateus, J. C. (2016). Abrumados y fascinados: Las TIC en la subjetividad de los docentes peruanos. Redes.com, (12), 171-193. https://doi.org/10.15213/re des.n12.p170

Mateus, J. C. \& Hernández-Breña, W. (2019). Design, validation, and application of a questionnaire on media education for teachers in training. Journal of New Approaches in Educational Research, 8(1), 34-41. http://hdl.handle. net/10230/32552

Mateus, J. C, Andrada, P. \& Quiroz (2020). Media Education in Latin America. Routledge. https://doi.org/10.4324/9780429244469

Mueller, J. \& Wood, E. (2012). Patterns of Beliefs, Attitudes, and Characteristics of Teachers That Influence Computer Integration. Education Research International, 2012, 1-13. https://doi.org/10.1155/2012/697357

PNUD. (2017). Desiguales. Orígenes, cambios y desafíos de la brecha social en Chile. Programa de las Naciones Unidas para el Desarrollo.

Raffaghelli, J. E. (2020). Generar actitudes digitales críticas en el alumnado. En A. Sangrà (coord.). Decálogo para la mejora de la docencia online. (pp. 169186). Editorial UOC. 
Rioseco, M. \& Roig, R. (2015). Expectativas que poseen los docentes universitarios de carreras de pedagogía en relación al uso de TIC. Píxel-Bit. Revista de Medios y Educación, 46, 51-64. https://www.redalyc.org/pdf/ 368/36832959011.pdf

Said, C. \& Gálvez, R. (8 de julio de 2020). La crisis golpea a universidades: aumenta deserción y 50 mil deudores del CAE piden apoyo. La Tercera. https:// www.latercera.com/nacional/noticia/la-crisis-golpea-a-universidades-au menta-desercion-y-50-mil-deudores-del-cae-piden-apoyo

Sang, G., Valcke, M., Braak, J. V. \& Tondeur, J. (2010). Student teachers' thinking processes and ICT integration: Predictors of prospective teaching behaviors with educational technology. Computers \& Education, 54(1), 103-112. https://doi.org/10.1016/j.compedu.2009.07.010

Tondeur, J., Braak, J. V., Ertmer, P. A. \& Ottenbreit-Leftwich, A. (2016). Understanding the relationship between teachers' pedagogical beliefs and technology use in education: a systematic review of qualitative evidence. Educational Technology Research and Development, 65(3), 555-575. https:// doi.org/10.1007/s11423-016-9481-2

Vicentini, I. C. (2020). La educación superior en tiempos de COVID-19: Aportes de la Segunda Reunión del Diálogo Virtual con Rectores de Universidades Líderes de América Latina. BID. http://dx.doi.org/10.18235/0002481 